\title{
Gout during the SARS-CoV-2 pandemic: increased flares, urate levels and functional improvement
}

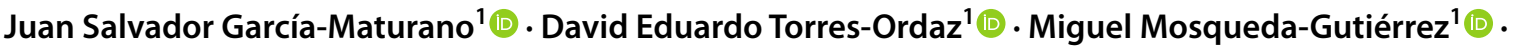

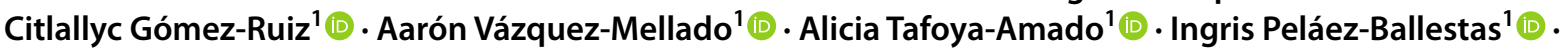 \\ Rubén Burgos-Vargas ${ }^{1}\left[\right.$ ] Janitzia Vázquez-Mellado ${ }^{1}$ A
}

Received: 14 June 2021 / Revised: 9 November 2021 / Accepted: 10 November 2021 / Published online: 25 November 2021

(C) International League of Associations for Rheumatology (ILAR) 2021

\begin{abstract}
Introduction Gout is the most common inflammatory arthritis, but was not considered in most COVID-19 and rheumatic diseases reports. Our aim was to describe changes in clinical data, treatment, function and quality of life for gout patients during COVID-19 pandemic.

Methods Prospective, descriptive and analytical study of 101 consecutive gout (ACR/EULAR 2015) patients from our clinic evaluated during pandemic by phone call $(n=52)$ or phone call + face-to-face $(n=68)$ that accepted to participate. Variables are demographics, clinical and treatment data, HAQ, EQ5D questionnaires and COVID-19-related data. Patients were divided in two groups: flare $(n=36)$ or intercritical gout $(n=65)$ also; available pre-pandemic data was obtained from 71 patients. Statistical analyses are $X^{2}$, paired $t$-test and Wilcoxon test.

Results Included gout patients were males (95.8\%), mean (SD) age 54.7 (10.7) years and disease duration 16.4 (9.8) years; $90 \%$ received allopurinol, $50 \%$ colchicine as prophylaxis and $25 \%$ suspended $\geq 1$ medication. Comparison of pre-pandemic vs pandemic data showed $>$ flares $(4.4 \%$ vs $36 \%, p=0.01)$, more flares in the last 6 months: $0.31(0.75)$ vs $1.71(3.1),(p=0.004$ and > urate levels: $5.6(1.7) v s 6.7(2.2) \mathrm{mg} / \mathrm{dL}, p=0.016$. Unexpectedly, function and quality-of-life scores improved: HAQ score $0.65(2.16)$ vs $0.12(0.17), p=0.001$. Seven patients were COVID-19-confirmed cases; they had significantly more flares, higher urate levels and lower allopurinol doses and two died.

Conclusions In gout patients, flares were 9 times more frequent during pandemic also, they had increased urate levels but led to an unexpected improvement in HAQ and functionality scores. Resilience and lifestyle changes in gout during COVID-19 pandemic require further studies.

\section{Key Points}

- COVID-19 pandemic is associated with 4 times more flares in gout patients.

- Increased flares were also seen in previously well-controlled gout patients.

- Increased serum urate levels were also found in gout patients during pandemic.

- In our gout clinic, 8/101 patients were diagnosed as COVID-19+, and two of them died.
\end{abstract}

Keywords Arthritis gouty · COVID-19 · Gout · Prognosis

The global disease burden of the novel coronavirus disease 2019 (COVID-19) has risen to levels unprecedented in our time. A World Health Organization (WHO) situation report in March 2021 presented global figures of 123,419,065 confirmed cases, 2,719,163 deaths and 393,531 new cases in the

Janitzia Vázquez-Mellado

jvazquezmellado@prodigy.net.mx

Extended author information available on the last page of the article previous $24 \mathrm{~h}$, with the Americas leading in both confirmed and new cases as well as in new deaths [1]. At this point, it is quite possible that the pandemic will become endemic despite all health measures implemented by the global community. It continues to be a complex and significant health issue that also affects the diagnosis and treatment of other diseases [2-4].

COVID-19 is a challenge for rheumatologists who need to analyse the direct and indirect impact of the SARS-CoV-2 
pandemic on rheumatic diseases [5]. Rheumatic patients are at higher risk of severe SARS-CoV-2 infection related to identifiable factors, including severity of the disease, smoking, ageing, comorbidities and treatment with immunosuppressants, particularly those with high doses of glucocorticoids [6-8]. Even so, although some reports conclude that rheumatic patients are three times more likely to be hospitalized in intensive care units or require mechanical ventilation, they do not have higher mortality rates [7-10].

Gout is the most common form of inflammatory arthritis [8], but in studies of COVID-19, musculoskeletal manifestations and rheumatic diseases, these patients were not considered to be at a greater risk of complications and mortality than those with systemic autoimmune rheumatic diseases [9-11].

Patients with gout have a higher risk of presenting comorbidities such as diabetes mellitus, hypertension, obesity and cardiovascular diseases [8], all of which are risk factors for higher mortality in COVID-19 [12].

Information about the incidence, risks, clinical expression and general morbidity and mortality of SARS-CoV-2 in gout is scarce [13]. A recent report included 4 patients with COVID-19, acute synovitis and previous diagnosis of gout or CPPD in whom confirmed crystals, but RT-PCR test negative for SARS-CoV-2 were found in synovial fluid [14], an internet survey for gout patients that evaluated characteristics of gout treatment, psychological distress and patient resilience during the pandemic [13] and recently the report of two cases of patients with gout who were on colchicine treatment and had SARS-CoV-2, both had mild symptoms [15].

Our objective in this study was to describe the characteristics and changes in clinical data (flares and intercritical gout), treatment modifications, functional variables and impact on quality of life during the pandemic and the frequency of COVID-19 in consecutive gout patients surveyed by phone call or phone call + face-to-face visit.

\section{Method}

\section{Gout clinic pre-pandemic}

The Gout Clinic of the Rheumatology Department in Hospital General de México has evaluated approximately 80 gout patients (first time and subsequent visits) per month in the last 20 years. This hospital is part of the public health care system, which provides partial coverage to the Mexican population. Most patients have a low socioeconomic status and frequently severe gout. Treatment is prescribed according to published recommendations or guidelines and medications available. Around half of the regular consecutive gout patients from the clinic are included in one of our gout projects (GRESGO cohort and severe gout) [16]. Patients are given a dedicated mobile phone number for gout patients, where they can leave instant or text messages if they have questions about their disease or medications and someone from the gout clinic contacts them within a few hours.

\section{Gout clinic during the pandemic}

From April to July 2020, the entire hospital was transformed into a COVID centre and all outpatient visits were cancelled. Research assistants made 2,393 phone calls to re-schedule rheumatic patients in order to give them information. The message was to "stay home, continue with the treatment and only go to the emergency room if necessary". At that time, 150 gout patients were called; 30 of them were not located, and 120 were located. During the phone call, gout patients were asked to participate in a "phone call visit" to answer several questions about their medications, health and functional status as well as COVID-associated questions about the patients themselves or their relatives. Gout patients could choose between going to the gout clinic in the weeks following for a face-to-face visit or to stay at home. Thirty patients refused the face-to-face visit due to fear of the virus or economic reasons (transportation expenses) and also because their diseases (gout and others) were under adequate control. Of the 120 patients located (68 phone call + face-to-face visit and 52 phone visit only), 101 agreed to participate in the study (Fig. 1).

\section{Gout evaluation}

We included gout (ACR/EULAR criteria) [17] patients that agreed to participate $(n=101)$ and were evaluated during the first year of the pandemic (March 2020 to February 2021). Based on the rheumatologist's evaluation at the time of the phone visit or phone + face-to-face visit, patients were divided into two groups: (1) flare $(n=36 / 101)$, determined according to published criteria [18] or (2) intercritical gout ( $n=65 / 101$ ) following the published definition [19]. None were considered to be in remission [20].

Eighty-one percent of the patients (82/101) accepted the face-to-face visit in the weeks following (Figure 1). Of these patients, we also had the clinical, biochemical and clinimetric data from the previous visit (pre-pandemic) of 71 gout patients who came to our clinic the year before. Patients whom agreed to participate signed the informed consent when they went to their next visit.

\section{Variable definitions}

During the phone call or face-to-face visits, we obtained demographic, clinical and treatment data related to gout 


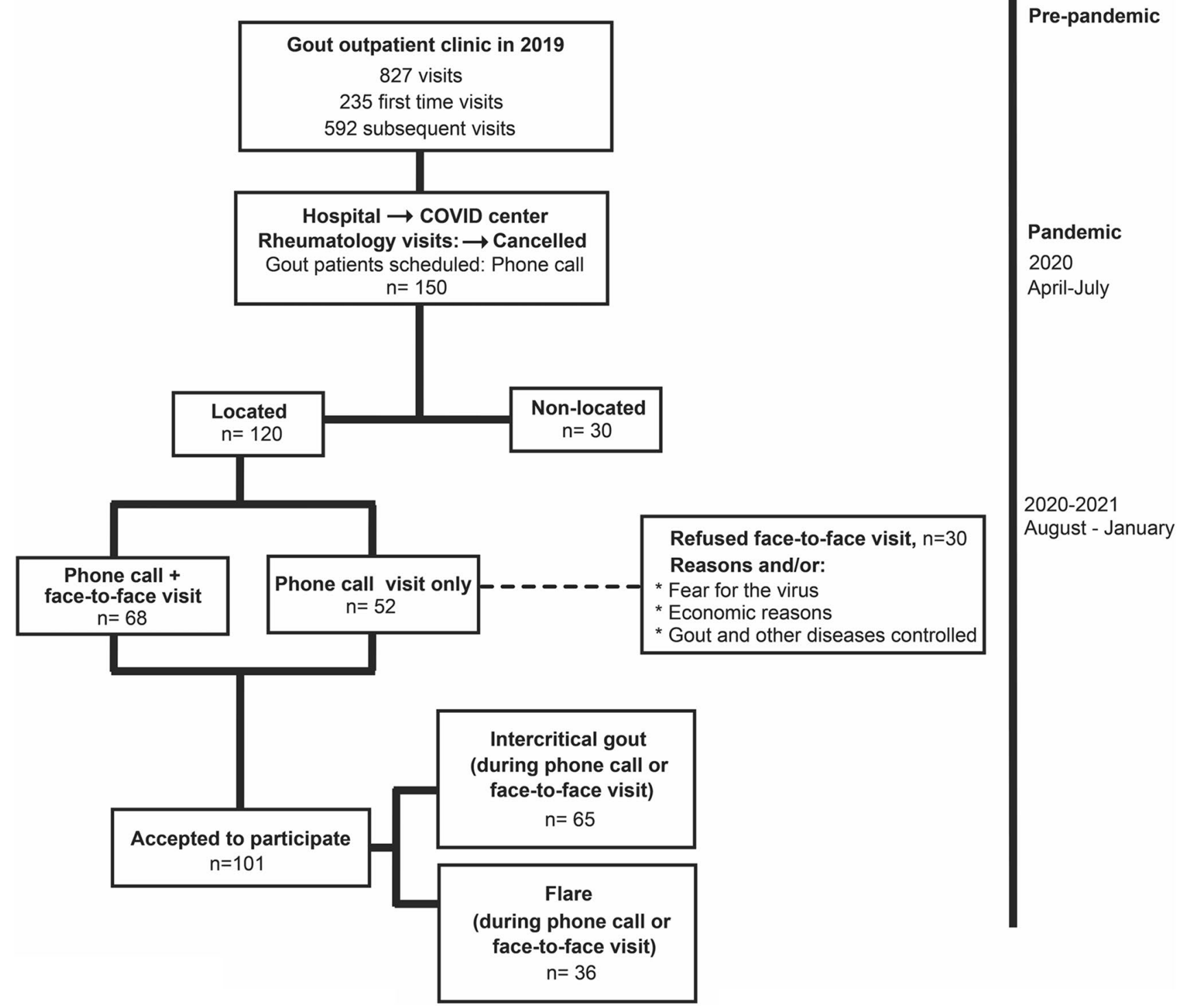

Fig. 1 Flow chart of the patients evaluated in the gout clinic during SARS-CoV-2 pandemic.

and associated diseases; metabolic syndrome and its components were considered according to ATP III criteria [21]. We applied Health Assessment Questionnaires (HAQ) [22] adapted for gout; Health-Related Quality of Life (HRQOL) [23] using the EQ-5D, these questionnaires are used regularly in our gout clinic; the patients answer the questionnaires during their regular visits. Joint count: number of tender, swollen and limited-motion joints [16] all these procedures were used in the pre-pandemic era as well as in pandemic. During pandemic visit, these variables were obtained from the patient themselves.

At regular visits, research assistants review with the patient the prescription from the last visit and the medications at the current visit; according to this information, we calculated the percentage of patients that suspended at least one of the prescribed medication.

\section{COVID-19 definitions}

Current operative definitions for SARS-CoV-2 were used (SI 1)

\section{Statistical analysis}

Descriptive data included mean (SD) or median and (IQR) when needed. $t$-test and chi-square test were used to compare flares vs intercritical gout patients. We also conducted a paired $t$-test or Wilcoxon test when nonparametric tests 
were required for the comparison between pre-pandemic vs pandemic data.

The project was authorized by the local IRB, and the patients signed informed consents (DI/20/404/03/58).

\section{Results}

This report included gout patients $(n=101)$ evaluated in our department over the last year during the SARS-CoV-2 pandemic. Most (95.8\%) were male, mean (SD) age 54.7 (10.7) years and with 16.4 (9.8) years' duration of the disease. Fifty-seven percent of them had tophaceous gout, $54 \%$ hypertriglyceridemia, $43 \%$ hypertension, $40 \%$ obesity, $16 \%$ diabetes and $14 \%$ hyperglycaemia. Most patients $(90 \%)$ received allopurinol as urate-lowering therapy (ULT), three received febuxostat and three probenecid (plus allopurinol) and 50\% received colchicine as prophylaxis. Twentyfive percent of the patients suspended at least one of their prescribed medications during the pandemic for economic reasons or because some medications were not available. There were no significant differences among patients that suspended medications vs those whom not (SI 2).
Flares vs intercritical gout patients at pandemic visit

During the pandemic, 36 patients (35\%) reported a recent or current flare, and $65 \%(p=0.01)$ reported intercritical gout during the phone or face-to-face evaluations. A comparison of patients with flares vs intercritical gout at the time of the visit found that patients with flares during the pandemic had several indicators of inadequate gout control (Table 1). We found no differences between patients evaluated by phone call vs phone call + face-to-face evaluation.

\section{Pre-pandemic vs pandemic evaluations for gout}

Pre-pandemic data from the previous visit to our clinic and the evaluation of the same patients during the pandemic were available for 71 of them. We considered and compared data from both evaluations (Table 2). According to pre-pandemic data from October 2019 to March 2020, 4.4\% of the patients had experienced a recent or current flare, and 95.6\% had intercritical gout at their regular evaluation in the gout clinic. As previously described, the percentage of patients with flares increased significantly during the pandemic $(p=0.01)$.
Table 1 Flares vs intercritical gout patients at pandemic visit
Table 2 Changes in gout patients during the SARS$\mathrm{CoV}-2$ pandemic

\begin{tabular}{llll}
\hline & Flares $(\mathrm{n}=36)$ & $\begin{array}{l}\text { Intercritical gout } \\
(\mathrm{n}=65)\end{array}$ & $\mathrm{p}$ \\
\hline Flares in the last 6 months, mean (SD) & $3.7(3.9)$ & $0.62(1.6)$ & 0.004 \\
median [IQR] & $2[1-11]$ & $0[0-5]$ & \\
Urate levels mg/dL, mean (SD) & $7.3(2.5)$ & $6.4(1.7)$ & 0.04 \\
Colchicine prophylaxis, \% & 33 & 54 & 0.001 \\
Allopurinol intake, \% & 93 & 90 & 0.60 \\
Allopurinol doses, mg; mean (SD) & $454.2(239.0)$ & $405(240)$ & 0.64 \\
Glucocorticoids (auto-prescribed), $(\%)$ & 37 & 8 & 0.0001 \\
\hline
\end{tabular}

\begin{tabular}{llll}
\hline & $\begin{array}{l}\text { Pre-pandemic evaluation } \\
n=71\end{array}$ & $\begin{array}{l}\text { Pandemic evaluation } \\
n=71\end{array}$ & $p^{\infty}$ \\
\hline Flare (\%) & $4.4^{\Omega}$ & 35 & 0.01 \\
Intercritical gout (\%) & $95.6^{\Omega}$ & 65 & \\
Flares last 6 months, median [IQR] & $0[0-1.4]$ & $1[0-10]$ & 0.004 \\
Urate mg/dL, mean (SD) & $5.6(1.7)$ & $6.7(2.2)$ & 0.016 \\
Limited to motion joints. median [IQR] & $0[0-10]$ & $0[0-3.1]$ & 0.005 \\
HAQ score, median [IQR] & $0.1[1-2.9]$ & $0[0-0.47]$ & 0.001 \\
Urate-lowering therapy (\%) & 93 & 90 & 0.30 \\
Allopurinol mg/day, mean (SD) & $472.5(262.3)$ & $404.8(244)$ & 0.36 \\
Colchicine, prophylaxis (\%) & 35 & 17 & 0.065 \\
BMI, mean (SD) & $29.4(4.9)$ & $29.7(4.9)$ & 0.86 \\
MDRD mL/min, mean (SD) & $76.2(28)$ & $69.9(25.7)$ & 0.51 \\
\hline
\end{tabular}

${ }^{\Omega}$ Percentage of patients evaluated as flares or intercritical gout from our cohort in the evaluations 6 months before March 16, 2020

${ }^{\ddagger}$ ULT: Allopurinol $n=90$, febuxostat $n=3 .{ }^{\infty}$ Paired $t$-test, Wilcoxon and chi-square test 
These patients had also significantly more flares and higher urate levels in the preceding months. Although not significant, a tendency toward higher BMI and lower renal function was observed in patients with flares. During the pandemic, patients reported better function; they improved significantly in functional data such as mean number of joints limited to motion (SD) (2 (4.2) vs 0.9 (2.7) $p=0.005)$ and mean HAQ score (SD) $(0.65$ (2.16) vs 0.12 $(0.17), \mathrm{p}=0.001)$ pre-pandemic vs pandemic evaluations, respectively.

Regarding EQ-5D pre-pandemic vs pandemic scores, we identified the percentage of patients with moderate or severe $(2+3)$ problems as follows: mobility $49 \%$ vs $21 \%, p=0.007$; self-care $21 \%$ vs $0 \%, p=0.001$; usual activities $36 \%$ vs $12 \%$, $p=0.005$; pain and discomfort $58 \%$ vs $34 \%, p=0.001$; and anxiety and depression $24 \%$ vs $8 \%, p=0.035$ decreased significantly (Table 2).

\section{COVID in gout patients and their relatives}

During the pandemic, eight gout patients from our gout clinic were diagnosed with suspected COVID-19, of which seven were confirmed, four of them received colchicine regularly as prophylaxis for flares and two died at home secondary to the disease, and one of them was on colchicine treatment (Table 3). During pandemic visit, gout patients with history of confirmed COVID-19 in the previous weeks or months had significantly more flares in the last 6 months, higher urate levels and lower allopurinol doses, when compared with gout patients without history of COVID-19 during pandemic (Table 4).

Only two patients reported employment problems due to the pandemic, although most patients in the clinic have irregular jobs. Nine relatives of the patients had suspected COVID-19. In terms of clinical data related to COVID-19, the most frequent manifestations in gout patients and their
Table 3 Regular treatment for gout in included patients during pandemic
Table 4 Gout patients and COVID-19

\begin{tabular}{lll}
\hline & $\begin{array}{l}\text { Pre-pandemic } \\
64 / 71\end{array}$ & $\begin{array}{l}\text { Pandemic } \\
88 / 100\end{array}$ \\
\hline ULT & & 88 \\
Allopurinol (\%) & 90 & $395.5(244.1)$ \\
Allopurinol, mg mean (SD) & $472.5(262.4)$ & 3 \\
Febuxostat (\%) & 2 & \\
Febuxostat, number of patients according to doses. & & 1 \\
40 mg/day & 0 & 1 \\
80 mg/day & 2 & 1 \\
120 mg/day & 0 & 0 \\
Probenecid, $n$ & 3 & 0 \\
500 mg, daily dose & 3 & $73(73)$ \\
Colchicine (prophylaxis), $n$ (\%) & $48(68)$ & $1.27(0.44)$ \\
Colchicine, mg: mean (SD) & $1.32(0.47)$ & 18 \\
$*$ Glucocorticoids (\%) & 4 & 7 \\
Prednisone, number of patients according to doses & & 1 \\
5 mg/day & 4 & 10 \\
10 mg/day & 0 & \\
Other glucocorticoids (auto-prescribed): Betamethasone or & 0 & \\
dexamethasone or methylprednisolone & & \\
\hline
\end{tabular}

\begin{tabular}{llll}
\hline & $\begin{array}{l}\text { Gout and COVID-19 } \\
n=7\end{array}$ & $\begin{array}{l}\text { Gout without COVID- } \\
19 \\
n=94\end{array}$ \\
\hline Flares, mean (SD) & $6.43(5.5)$ & $1.35(2.7)$ & 0.008 \\
Urate levels, mean (SD) & $8.6(2.0)$ & $6.62(2.4)$ & 0.044 \\
Allopurinol, doses mean (SD) & $150(229)$ & $413(236)$ & 0.005 \\
Death & $2 / 7$ & $0 / 94$ & 0.0001 \\
\hline
\end{tabular}


relatives were fever, headache, cough and dyspnoea; none of the gout patients had flare during COVID-19.

\section{Discussion}

At our gout clinic, we observed an increased percentage of flares vs intercritical gout patients in our phone and face-toface evaluations. This was confirmed when we compared the evaluations of the same patients before and during the pandemic, where we observed nearly nine times more flares in our gout patients evaluated in this period. These patients also had significantly more flares in the last months, higher urate levels and, although not significant, higher BMI, lower renal function and changes in their treatment such as suspending all medications for one or several days and/or modifying the doses; in addition the entire hospital was transformed into COVID centre, and all outpatient visits were cancelled for several months. Moreover, seven patients with gout had confirmed COVID-19 in the previous months to visit, in them we found data of uncontrolled gout (more flares, higher urate levels and lower allopurinol doses). Two of them died at home.

Previous studies have evaluated metabolic impact during lock-down and focused on changes in lipids, glucose, transaminases, TNF-alpha and acute phase reactants, but urate levels during the COVID-19 pandemic had not been evaluated previously [24]. The pandemic implied a generalized lock-down that definitely changed diet, exercise and lifestyle habits. Furthermore, previous studies demonstrated boredom and increased caloric intake particularly of carbohydrates secondary to limitations in obtaining fresh fruits and vegetables during the pandemic [25]. While all of these are possible explanations, we did not evaluate lifestyle changes in this paper.

We also found unexpected significant improvement in functional and quality-of-life variables. Some previous reports found increased self-care and physical activity (time and quality). A report from Belgium [25] showed that 58\% of people who previously had been sedentary or with low physical activity exercised more, $5 \%$ the same and $7 \%$ less than pre-pandemic; $61 \%$ of them reported having more time for exercise. Another study evaluating gout itself, quality of life, psychological distress and patient resilience during the pandemic through an online survey found few worse scores related to the COVID-19 pandemic [13]. These differences could be related to varying socioeconomic and educational levels, cultural beliefs, resilience [26] and the fact that our report includes regular patients from the gout clinic who felt at ease with us during the phone or face-to-face visits and who had previous experience in answering EQ 5D or HAQ questionnaires at their regular visits.
There are several limitations to our study to be considered. We could only locate $80 \%$ of the pre-pandemic patients with scheduled visits. Non-located gout patients from our clinic are those with lower educational level, health status and severe gout [16] frequently associated with chronic autoprescribed glucocorticoids; these severities are probably not seen in other centres or countries. We did not evaluate lifestyle changes and resilience.

According to official data [27, 28], 3,431,073 COVID-19 cases including 266,003 deaths had been reported in Mexico as of August 23, 2021, with a fatality rate of $7.7 \%$ for the general population. In this report from our gout clinic, we included 101 patients, of whom eight (7.9\%) were COVID19 suspect cases, seven confirmed cases and two deaths, resulting in a fatality rate of $19.8 \%$. A previous report from our country included 164 COVID-19 patients, intubated at ICU in 11 hospitals in Mexico; these patients had severe acute respiratory syndrome (SARS-CoV-2); the mortality rate was 51.8\% during April 2020 [29]. These data could be compared to developed countries whose mortality was lower $(30.5 \%)$ in patients with similar conditions [30]. This could be due to the fact that patients in our country arrive at emergency room with higher SOFA and APACHE scores than in developed countries; other possible associated factors are population density, larger households and the frequency of long-term conditions such as hypertension, diabetes and obesity, already known to be associated with increased risk for mortality [31].

Clearly, the pandemic brought many changes worldwide, in our country and in our gout clinic. We found increased numbers of flares closely associated with higher urate levels, treatment modifications and unexpected functional improvement. The impact of COVID-19 on several diseases and mortality will undoubtedly be the subject of further studies.

Code availability No applicable

Funding The gout clinic receives unrestricted partial financial support from the Dirección de Investigación of the Hospital General de México.

Data availability Data and material of this project is available for revision if requested.

\section{Declarations}

Ethics approval The project has been approved by the Research Ethics Committee of the Hospital General de México Dr. Eduardo Liceaga (DI/20/404/03/58). Informed consent for the use and publication of the survey data was obtained from all participants.

Consent to participate and publication All authors agreed to participate and authorized the final version of the manuscript sent to Clin Rhematol.

Disclosures None. 


\section{References}

1. WHO Coronavirus Disease (COVID-19) Dashboard. Available at: https://covid19.who.int/ [Internet Accessed March 23, 2021.]

2. Cobey S (2020) Modeling infectious disease dynamics. Science 368(6492):713-714. https://doi.org/10.1126/science.abb5659

3. Ibáñez B (2020) Myocardial infarction in times of COVID-19. Rev Esp Cardiol. 73:975-977. https://doi.org/10.1016/j.recesp. 2020.09.022

4. Rodríguez-Leor O, Cid-Álvarez B, Pérez de Prado A, Rossello X, Ojeda S, Serrador A et al (2020) Impact of COVID-19 on ST-segment elevation myocardial infarction care. The Spanish experience. Rev Esp Cardiol (Engl Ed). 73:994-1002. https:// doi.org/10.1016/j.recesp.2020.07.033

5. Morales-Torres J, Aceves-Ávila FJ (2020) Rheumatologists in the COVID-19 era: will there be a new role for the rheumatologist in the care of rheumatic patients? Clin Rheumatol 39:3177-3183. https://doi.org/10.1007/s10067-020-05380-1

6. Akiyama S, Hamdeh S, Micic D, Sakuraba A (2021) Prevalence and clinical outcomes of COVID-19 in patients with autoimmune diseases: a systematic review and meta-analysis. Ann Rheum Dis. 80:384-391. https://doi.org/10.1136/annrh eumdis-2020-218946

7. Strangfeld A, Schäfer M, Gianfrancesco MA, Lawson-Tovey S, Liew JW, Ljung L et al (2020) Factors associated with COVID19-related death in people with rheumatic diseases: results from the COVID-19 Global Rheumatology Alliance physician-reported registry. Ann Rheum Dis -219498. https://doi.org/10.1136/annrh eumdis-2020-219498

8. Singh JA, Gaffo A (2020) Gout epidemiology and comorbidities. Seminars in Arthritis and Rheumatism 50:11-16. https://doi.org/ 10.1016/j.semarthrit.2020.04.008

9. Gianfrancesco M, Hyrich KL, Al-Adely S, Carmona L, Danila MI, Gossec L et al (2020) Characteristics associated with hospitalization for COVID-19 in people with rheumatic disease: data from the COVID-19 Global Rheumatology Alliance physician-reported registry. Ann Rheum Dis. 79:859-866. https://doi.org/10.1136/ annrheumdis-2020-217871

10. Hyrich KL, Machado PM (2021) Rheumatic disease and COVID19: epidemiology and outcomes. Nat Rev Rheumatol. 17:71-72. https://doi.org/10.1038/s41584-020-00562-2

11. D'Silva KM, Serling-Boyd N, Wallwork R, Hsu T, Fu X, Gravallese EM et al (2020) Clinical characteristics and outcomes of patients with coronavirus disease 2019 (COVID-19) and rheumatic disease: a comparative cohort study from a US 'hot spot'. Ann Rheum Dis. 79:1156-1162. https://doi.org/10.1136/annrh eumdis-2020-217888

12. Li B, Yang J, Zhao F, Zhi L, Wang X, Liu L et al (2020) Prevalence and impact of cardiovascular metabolic diseases on COVID19 in China. Clin Res Cardiol. 109:531-538. https://doi.org/10. 1007/s00392-020-01626-9

13. Singh JA, Edwards NL (2020) Gout management and outcomes during the COVID-19 pandemic: a cross-sectional internet survey. Ther Adv Musculoskelet Dis. 12:1759720x20966124. https://doi. org/10.1177/1759720X20966124

14. López-González MD, Peral-Garrido ML, Calabuig I, TovarSugrañes E, Jovani V et al (2020) Case series of acute arthritis during COVID-19 admission. Ann Rheum Dis. 2020; annrheumdis-2020-217914. https://doi.org/10.1136/annrh eumdis-2020-217914

15. Pelechas E, Drossour V, Voulgari PV et al (2021) COVID-19 in patients with gout on colchicine. Rheumatol Int 41:1503-1507. https://doi.org/10.1007/s00296-021-04902-7
16. Alvarado-de la Barrera C, López-López CO, Álvarez-Hernández E, et al. (2020) Are target urate and remission possible in severe gout? A five-year cohort study. J Rheumatol. 2020;47:132-139 https://doi.org/10.3899/jrheum.181214

17. Neogi T, Jansen TL, Dalbeth N, Fransen J, Schumacher HR, Brensen D et al (2015) (2015) 2015 gout classification criteria: an American College of Rheumatology/European League Against Rheumatism collaborative initiative. Arthritis Rheumatol 67:2557-2568. https://doi.org/10.1002/art.39254

18. Gaffo AL, Dalbeth N, Saag KG, Singh JA, Rahn EJ, Mudano AS et al (2018) (2018) Brief report: validation of a definition of flare in patients with established gout. Arthritis Rheumatol 70:462-467. https://doi.org/10.1002/art.40381

19. Bursill D, Taylor WJ, Terkeltaub R et al (2019) (2019) Gout, hyperuricemia, and crystal-associated disease network consensus statement regarding labels and definitions for disease elements in gout. Arthritis Care Res. 71:427-434. https://doi.org/10.1002/acr. 23607

20. Chapman P et al (2016) (2016) Development of preliminary remission criteria for gout using Delphi and 1000 Minds consensus exercises. Arthritis Care Res 68:667-672. https://doi.org/10. 1002/acr.22741

21. Andrés M, Bernal JA, Sivera F et al (2016) Cardiovascular risk of patients with gout seen at rheumatology clinics following a structured assessment. Ann Rheum Dis.2017;76:1263-1268. https:// doi.org/10.1136/annrheumdis-2016-210357

22. Alvarez-Hernandez E, Peláez-Ballestas I, Vazquez-Mellado J, Terán-Estrada L, Bernard Medina AG, Espinoza J et al (2008) Validation of the health assessment questionnaire disability index in patients with gout. Arthritis Rheum 15:665-669. https://doi. org/10.1002/art.23575

23. Herdman M, Badía X, Berra S (2001) (2001) EuroQol-5D: a simple alternative for measuring health-related quality of life in primary care. Aten Primaria 28:425-430. https://doi.org/10.1016/ s0212-6567(01)70406-4

24. Martinez-Ferran M, de la Guía-Galipienso F, Sanchis-Gomar F, Pareja-Galeano H (2020) Metabolic impacts of confinement during the COVID-19 pandemic due to modified diet and physical activity habits. Nutrients. 2020:12. https://doi.org/10.3390/nu120 61549

25. Constandt B, Thibaut E, De Bosscher V, Scheerder J, Ricour M, Willem A (2020) Exercising in times of lockdown: an analysis of the impact of COVID-19 on levels and patterns of exercise among adults in Belgium. Int J Environ Res Public Health. 2020:17(11). https://doi.org/10.3390/ijerph17114144

26. Walsh F (2020) (2020) Loss of resilience in the time of COVID19: Meaning making, hope and transcendence. Fam Proc 59:898911. https://doi.org/10.1111/famp. 12588

27. Salud Sd. Informes Epidemiológicos de la Situación de COVID19 en México. In: Documentos, editor: Secretaría de Salud; 2021. Available at: https://www.gob.mx/salud/documentos/coronaviruscovid-19-comunicado-tecnico-diario-238449 [Internet Accessed August 3, 2021.

28. Public health surveillance for COVID-19; WHO-2019-nCoVSurveillanceGuidance-2020.8. (Internet. Accessed March 28, 2021) Available from: https://www.who.int/publications/i/item/ who-2019-nCoV-surveillanceguidance-2020.8

29. N amendys-Silv Silvio A, Alvarado-Avila Pedro A., DominguezCherit Guillermo, Rivero-Sigarroa Eduardo Sanchez-Hurtado Luis Et al (2020) Outcomes of patients with COVID-19 in the Intensive Care Unit in Mexico: a multicenter observational study, Elsevier, Heart-Lung, 10-20-2020 https://doi.org/10.1016/j.hrtlng. 2020.10.013 
30. COVID-ICU Group on behalf of the REVA Network and the COVID-ICU Investigators(2021) Clinical characteristics and day-90 outcomes of 4244 critically ill adults with COVID-19: a prospective cohort study, Springer,Intensive Care Med, 2021 https://doi.org/10.1007/s00134-020-06294-x

31. Joy Mark, Hobbs Richard, Lopez Bernal J., Sherlock Julian, Amirthalingam Gayatri, McGagh Dylan et al (2020) Excess mortality in the first COVID pandemic peak: cross-sectional analyses of the impact peak: cross-sectional analyses of the impact of age, sex, ethnicity, household size, and long-term conditions in people of known SARS-CoV-2 status in England, British Journal of General Practice, December 2020 of an abridged version published inprint. Cite this version as: Br J Gen Pract 2020; https://doi.org/ 10.3399/bjgp20X713393

Publisher's note Springer Nature remains neutral with regard to jurisdictional claims in published maps and institutional affiliations.

\section{Authors and Affiliations}

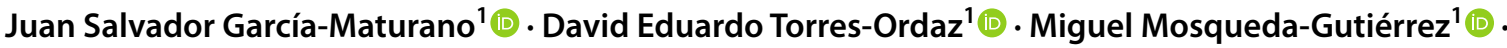

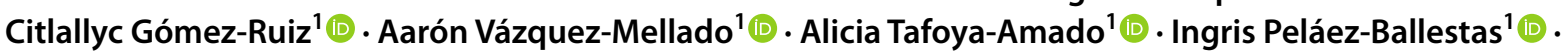 Rubén Burgos-Vargas ${ }^{1}$ (1) . Janitzia Vázquez-Mellado ${ }^{1}$ (i)}

Juan Salvador García-Maturano

juansgam93@gmail.com

David Eduardo Torres-Ordaz

411davidtorres@gmail.com

Miguel Mosqueda-Gutiérrez mamg0118@gmail.com

Citlallyc Gómez-Ruiz citlallyc.ruiz@gmail.com

Aarón Vázquez-Mellado aaronvmm@hotmail.com
Alicia Tafoya-Amado

psaliciatafoya@gmail.com

Ingris Peláez-Ballestas pelaezin@gmail.com

Rubén Burgos-Vargas

r.burgos.vargas@gmail.com

1 Servicio de Reumatología, Unidad 404, Hospital General de México Dr. Eduardo Liceaga Dr. Balmis 148, Col. Doctores, C.P. 06720 Mexico City, Mexico 